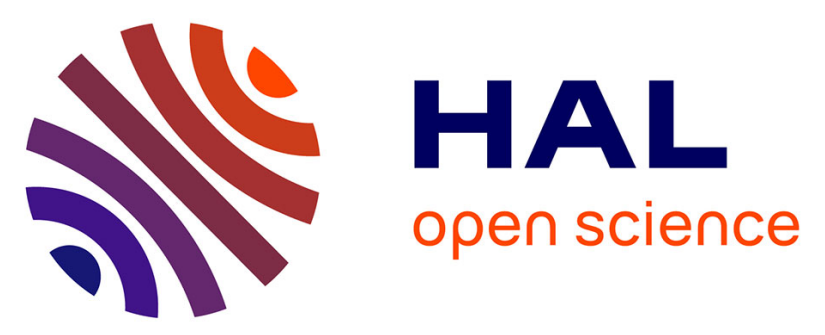

\title{
Spatio-temporal structure of femtosecond Bessel beams from spatial light modulators
}

\author{
Luc Froehly, Maxime Jacquot, Pierre-Ambroise Lacourt, John Michaël \\ Dudley, François Courvoisier
}

\section{To cite this version:}

Luc Froehly, Maxime Jacquot, Pierre-Ambroise Lacourt, John Michaël Dudley, François Courvoisier. Spatio-temporal structure of femtosecond Bessel beams from spatial light modulators. Journal of the Optical Society of America. A Optics, Image Science, and Vision, 2014, 31 (4), pp.790-793. 10.1364/JOSAA.31.000790 . hal-03222049

\section{HAL Id: hal-03222049 \\ https://hal.science/hal-03222049}

Submitted on 10 May 2021

HAL is a multi-disciplinary open access archive for the deposit and dissemination of scientific research documents, whether they are published or not. The documents may come from teaching and research institutions in France or abroad, or from public or private research centers.
L'archive ouverte pluridisciplinaire HAL, est destinée au dépôt et à la diffusion de documents scientifiques de niveau recherche, publiés ou non, émanant des établissements d'enseignement et de recherche français ou étrangers, des laboratoires publics ou privés. 


\title{
Spatio-temporal structure of femtosecond Bessel beams from spatial light modulators
}

\author{
L. Froehly ${ }^{*}$, M. Jacquot, P. A. Lacourt, J. M. Dudley and F. Courvoisier \\ FEMTO-ST Institute, Department of Optics P.M. Duffieux, UMR CNRS 6174, Université de Franche-Comté, \\ 25030 Besançon, France \\ *Corresponding author: 1uc.froehly@univ-fcomte.fr
}

Received Month X, XXXX; revised Month X, XXXX; accepted Month X, XXXX; posted Month X, XXXX (Doc. ID XXXXX); published Month X, XXXX

\begin{abstract}
We numerically investigate the spatio-temporal structure of Bessel beams generated with Spatial Light Modulators (SLMs). Grating-like phase masks enable the spatial filtering of undesired diffraction orders produced by SLMs. Pulse front tilt and temporal broadening effects are investigated. In addition, we explore the influence of phase wrapping and show the spatio-temporal structure of SLM-generated femtosecond Bessel beams is similar to Bessel X-pulses at short propagation distance, and to subluminal Pulsed Bessel Beams at long propagation distance.
\end{abstract}

\section{Introduction}

Non-diffracting Bessel beams constitute a class of solutions to the propagation equation that intensity profile is invariant along the propagation direction [1]. A zero-order Bessel beam is an axisymmetric interference of plane waves propagating at an angle $\theta$ with respect to the optical axis (referred as the conical angle), that generates an intense central spot surrounded by several lobes of lower intensity. These beams have been mainly used for optical manipulation [2,3], microscopy [4] and also provide novel perspectives for localized waves [5], and ultrashort pulse filamentation [6]. The stationarity property of Bessel beams in the nonlinear propagation regime [7] has been used to overcome many of the difficulties of high aspect ratio laser micro- and nano- structuring of metals and dielectrics [8-10].

The spatio-temporal shape of the femtosecond Bessel beam is of crucial importance during the propagation of the pulse in nonlinear media. Two kinds of Bessel wavepackets generated from ultrashort laser pulses and limited bandwidth in normal dispersion media are i)Bessel X-pulses (BXP) and ii) Pulsed Bessel beams. BXP result from the superposition of monochromatic Bessel beams with identical 
conical angle $\theta$ and a variable transverse wavevector in vacuum $k_{\perp}=(\omega / c) \sin \theta$. They can be generated with axicons and exhibit a superluminal on-axis group velocity $v_{g}=c / \cos \theta$ [11]. The superluminal behavior does not violate physical laws: no information is propagated along the optical axis because of the conical structure of the waves [11]. Pulsed Bessel beams (PBB) have a constant transverse wavevector and therefore a variable conical angle. They are generated by flat holograms and have a sub-luminal group velocity $v_{g}=c \cos \theta$, where $\theta$ is the conical angle at the central frequency of the pulse [12,13]. The ability of freely designing phase masks with Spatial Light Modulators (SLMs) makes them an attractive solution to generate femtosecond Bessel beams [14-16]. Recently, the spatio-temporal structure of localized wavepackets generated by SLMs has been studied experimentally and theoretically $[17,18]$. However, the effect of the phase wrapping on the spatio-temporal dynamics of femtosecond Bessel beams generated by SLMs was not investigated.

Here, we perform numerically this investigation on the basis of an experimental setup that generates high-quality micron-size femtosecond Bessel beams. We model the complete setup including Fourier filtering and report in which conditions the spectral content and the pulse duration of the pulse is preserved. We numerically reconstruct the propagation in space and time of Bessel pulses and highlight that their structure is close to Bessel X Pulses at short propagation distance and Pulsed Bessel Beams at longer distance.

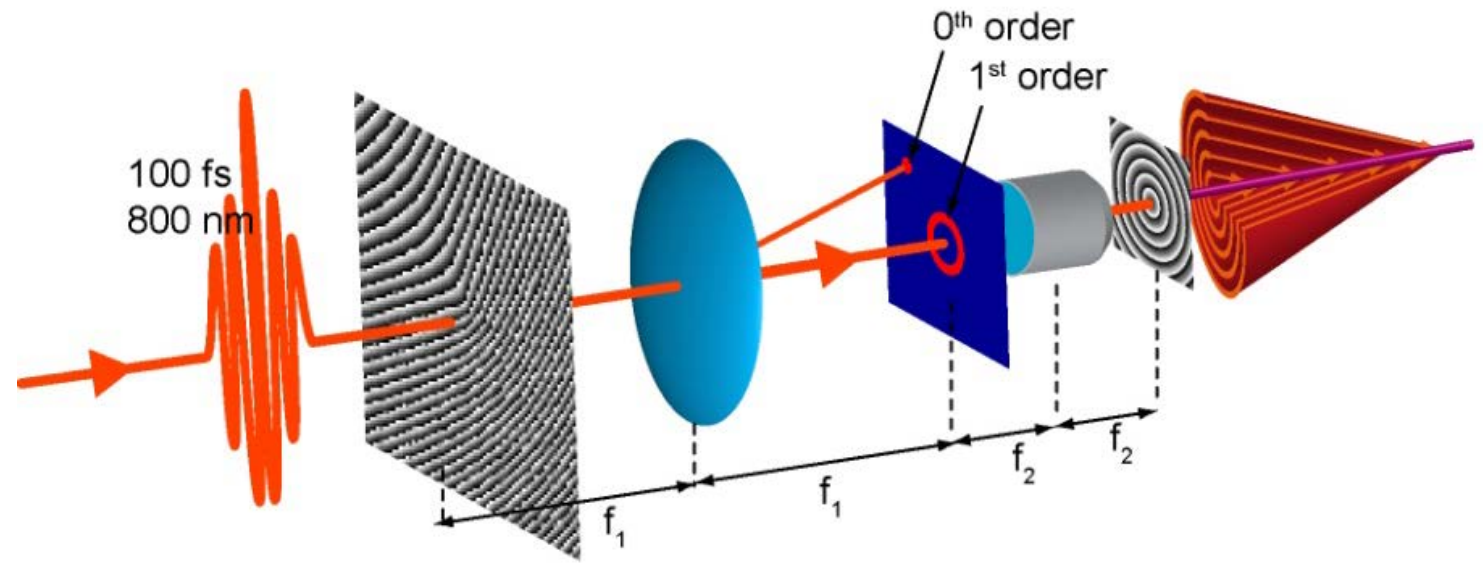

Fig. 1. Experimental setup for femtosecond Bessel beam generation. A 100fs laser beam is incident onto an SLM on which is encoded a phase mask Spatial filtering is performed in the Fourier plane of the first lens of the demagnification system. Lens and microscope objective form a 4-f demagnification system to increase the range of achievable conical angles.

The experimental setup for SLM-based femtosecond Bessel beam generation is shown on figure 1. The laser source is an amplified 100 fs Ti:Sa laser with central wavelength $800 \mathrm{~nm}$. The optically-adressed SLM (Hamamatsu PAL-SLM [19]) imprints on the Gaussian laser beam a computer-controlled phase mask similar to an axicon and that is detailed in the next paragraph. A lens with focal distance $\mathrm{f}_{1}=1 \mathrm{~m}$ and a microscope objective $\left(\mathrm{MO}, \mathrm{f}_{2}=3.6 \mathrm{~mm}\right)$ are set in a 4 -f configuration. Spatial filtering with an iris is performed in the common focal plane of the lens and the MO. Therefore, the field generated by the SLM phase mask is Fourier-filtered and imaged on 
the focal plane of the MO, and the magnification factor is $\mathrm{f}_{2} / \mathrm{f}_{1}$. We will show in the following how the magnification allows us to generate micro-Bessel beams with high conical angle that onset from the MO focal plane.

In a Spatial Light Modulator, a layer of liquid crystals with thickness $T$ is electrically or optically addressed to modify its refractive index $n(x, y)$ [19]. In our case, for a reflective SLM, the optical path length is 2T.n(x,y) and the phase applied on the beam, after propagation through the SLM, depends on the frequency $\omega$ as: $\Phi(x, y)=2 T n(x, y) \omega / c$. We note that the intrinsic dispersion of the liquid crystal is negligible for pulses down to $\sim 10 \mathrm{fs}$ at $800 \mathrm{~nm}$ central wavelength. To generate a Bessel beam from a Gaussian one, we apply a rotationally symmetric index change $n(x, y)=r \sin (\theta) /(2 T)$, with $r=\sqrt{x^{2}+y^{2}}$. The phase mask then reads as: $\Phi(x, y, \omega)=(\omega / c) r \sin \theta$. This produces a Bessel beam with a radial component of the wavevector $k_{r}=k \sin \theta$ where $k=\omega / c$ is the wavevector. Importantly, the conical angle $\theta$ is independent of the frequency, in contrast with reference [20] where the phase was supposed to be invariant with the frequency.

At wavelengths $\sim 800 \mathrm{~nm}$, the phase modulation depth of the SLM is limited to $\sim 3 \pi$. Therefore, the phase mask at the central frequency is wrapped between 0 and $2 \pi$. The wrapping however produces undesired diffraction orders. To spatially separate them and allow for spatial filtering, we add a linear phase shift in the $(x y)$ plane that produces a blazed grating. Finally, the phase mask reads as:

$$
\Phi(\vec{r}, \omega)=\frac{\omega}{\omega_{0}} \Phi\left(\vec{r}, \omega_{0}\right)=\frac{\omega}{\omega_{0}}\left[\frac{\omega_{0}}{c} \mathrm{rsin} \theta+\overrightarrow{k_{1}} \cdot \vec{r} \quad[2 \pi]\right]
$$

where $\overrightarrow{k_{1}}=\frac{\omega_{0}}{c} \sin \alpha \vec{u}$ with $\vec{u}$ a unitary vector in the SLM plane, and $\alpha$ is the deviation angle of the +1 diffracted order.

In the focal plane of L1, the first order of diffraction of the beam at a frequency $\omega$ is an annulus of radius $R=f_{1} \sin \theta$. Due to the grating-like structure induced by the second term $\overrightarrow{k_{1}} \cdot \vec{r}$ of the phase mask (Eq. (1)), the different frequencies are angularly dispersed and the centers of the annuli are distributed along the axis $\vec{u}$. The optical axis of the microscope objective is centered on the central frequency. Finally, after Fourier-transforming the beam with the microscope objective, each input frequency is shaped into a Bessel beam with a conical angle $\theta^{\prime}=\theta f_{1} / f_{2}$ and that propagates along an axis tilted from the optical axis by an angle $\gamma(\lambda)=-\frac{f_{1}}{f_{2}} \frac{\left(\lambda-\lambda_{0}\right)}{\lambda_{0}} \sin \alpha$. The demagnification factor $f_{1} / f_{2}$ allows us to largely increase the values of achievable conical angles $\theta^{\prime}$ after the microscope objective (up to $26^{\circ}$ in our experimental setup) .A transmission efficiency of $\sim 25 \%$ in the first order of diffraction has been experimentally measured in our configuration. 

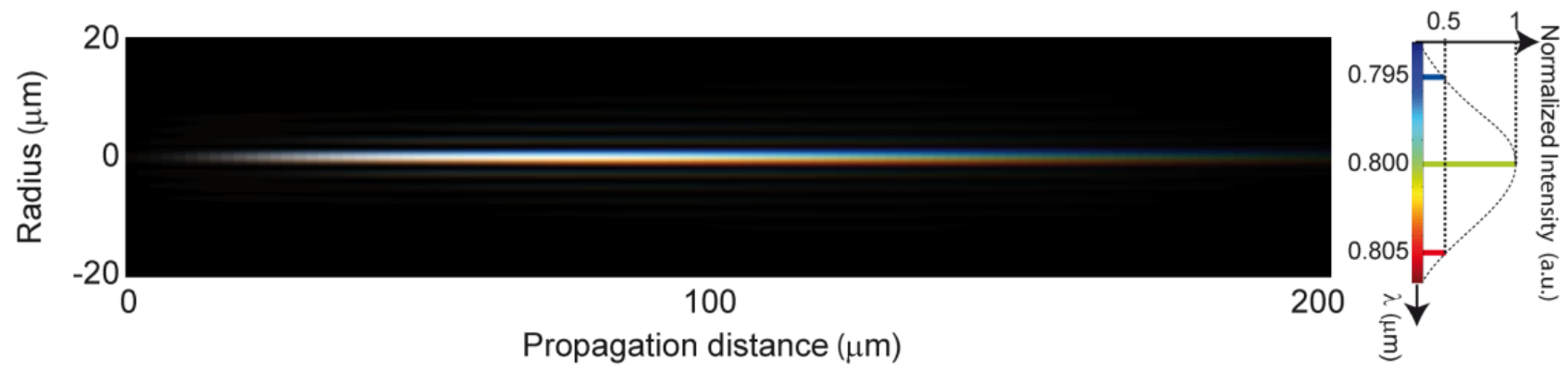

Fig. 2. Intensity map I(z,r) for three different wavelengths covering the spectral FWHM of a $100 \mathrm{fs}$ laser beam encoded on 3 color channels. The white regions show clearly where the spectral content of the beam is homogeneous. This shows the effect of the small angular dispersion $\left(\gamma \leq 1^{\circ}\right)$.

Figure 2 shows the effect of the spectral dispersion $\gamma(\lambda)$. We superposed the intensity maps at three different wavelengths covering the spectral range FWHM of a 100 fs laser (795 nm in blue, $800 \mathrm{~nm}$ in green and $805 \mathrm{~nm}$ in red). Each intensity distribution was encoded in one of the RGB channels so that the regions in space where the beams overlap appear in white. The intensity distributions are linearly tilted by an angle $\gamma(\lambda)$ from the optical axis, but a good overlap is observed almost all along the beam. Indeed, the diffraction-free range of a Bessel beam is $z_{\max } \approx w_{0}{ }^{\prime} / \sin \theta^{\prime}[2]$ where $w_{0}{ }^{\prime}=w_{0} f_{2} / f_{1}$ is the waist of the image of the Gaussian beam with waist $w_{0}$ that illuminates the SLM. The radius of the central lobe is: $r_{0}(\lambda)=2.405 \lambda /\left(2 \pi \sin \theta^{\prime}\right)$. Therefore, the lateral shift of the beam over the Bessel zone, $z_{\max } \gamma\left(\lambda_{0}+\Delta \lambda\right)$ at a wavelength $\lambda_{0}+\Delta \lambda$ is smaller than the radius of the central lobe if $\Delta \lambda \leq 2.405 \lambda_{0}^{2} /(2 \pi w \sin \alpha)= \pm 14 \mathrm{~nm}$ in our case $(\alpha=5.3 \mathrm{mrad}, w=3.2 \mathrm{~mm})$. Therefore, the influence of the grating structure on the intensity distribution is negligible for $100 \mathrm{fs}$ pulses, independently of the conical angle.

Figure 3 (a) compares the experimental and numerical intensity distributions of a $100 \mathrm{fs}$ Bessel beam with a conical angle $\theta^{\prime}=26^{\circ}$. The distribution is shown in the plane defined by the optical axis and the vector $\vec{u}$ where the lateral shift is the highest. In our numerical model, the field of a 100 fs pulse is propagated from the SLM plane to the MO focal plane with appropriate Fourier transforms and filtering; in a second step it is propagated from the MO focal plane, along the Bessel zone, with the nonparaxial angular spectrum of plane waves [21]. The comparison with the experiment shows an excellent agreement. Figure 2(b) shows the experimental cross section of the beam. Here the central spot size is $0.7 \mu \mathrm{m}$ FWHM.

a)

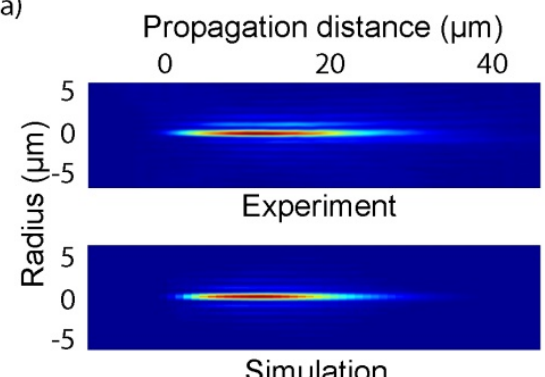

Simulation b)

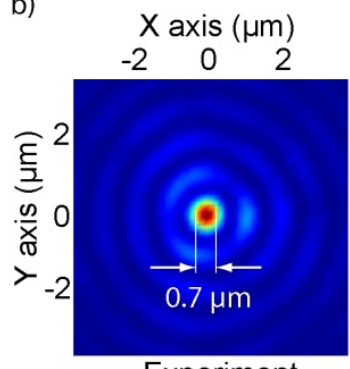

Experiment 
Fig. 3. (a) Comparison of the experimental and numerical intensity maps in the $(r, z)$ plane for a 100 fs Bessel beam with conical angle of $26^{\circ}$

Corresponding experimental beam cross section.

\section{Spatio-temporal analysis}

\section{A.Pulse front tilt and temporal broadening}

Although the influence of the grating structure of the SLM is negligible on the intensity distribution, it generates a pulse front-tilt on the Bessel pulse. We have reconstructed the spatio-temporal linear propagation of Bessel pulses for a conical angle $\theta^{\prime}=5^{\circ}$. Figure 4 shows the temporal beam evolution at different propagation distances $\mathrm{z}$ of the numerically reconstructed laser pulse. Importantly, we observe (Figure $4(\mathrm{a})$ ) the presence of a temporal broadening that is less than $10 \%$ between $\mathrm{z}=0$ and $\mathrm{z}=200 \mu \mathrm{m}$. This temporal broadening is a consequence of the grating like effect due to the linear phase shift. Due to spectral dispersion, a pulse-front tilt $\Delta$ is also visible (Figure $4(\mathrm{~b}))$ and expresses as: $\Delta=-\lambda \partial \gamma / \partial \lambda=\sin \alpha f_{1} / f_{2}=0.55 \mathrm{rad}$ [22] with $\tan \Delta=\tan \Delta_{\tau} / c$. The lobes of the Bessel beam are still parallel to the optical axis, but the upper side is retarded compared to the lower one.

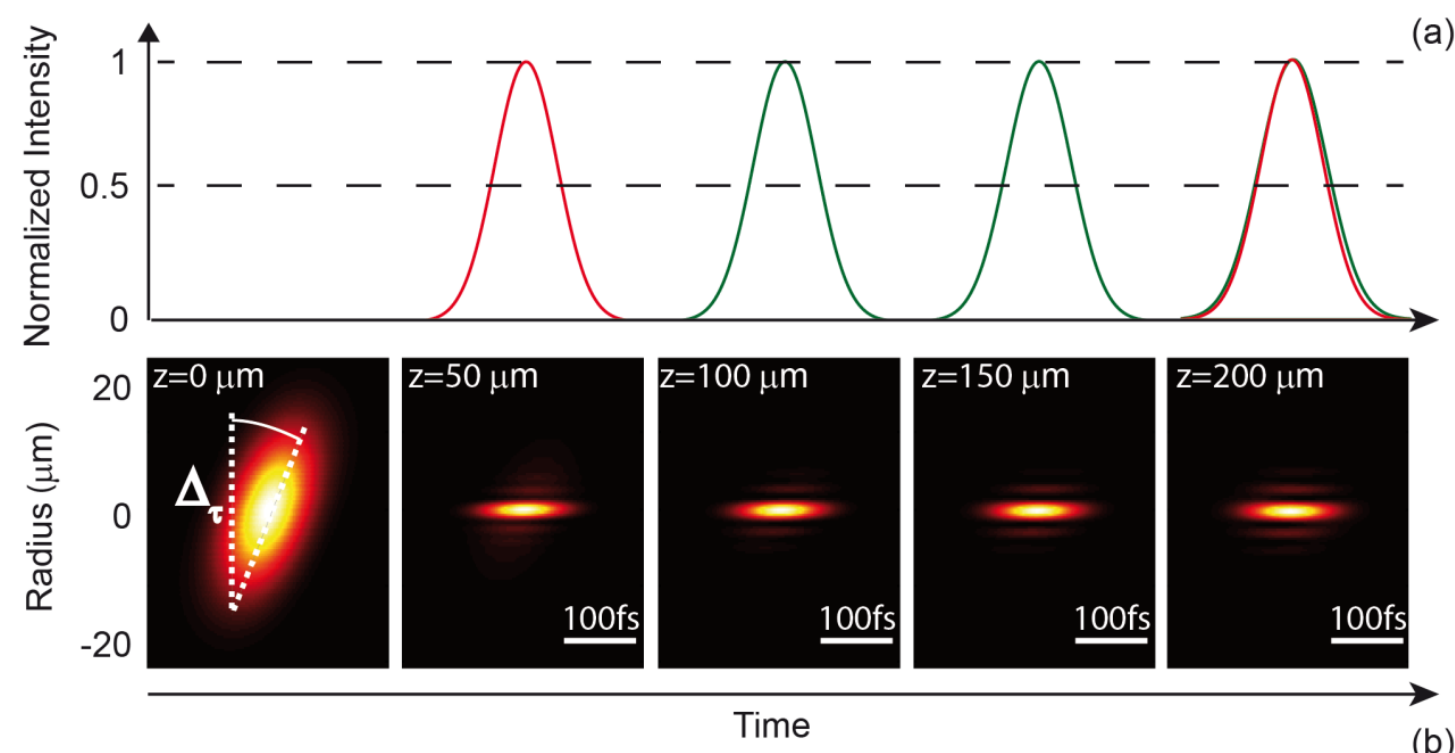

Fig. 4. Normalized intensity map of the pulse I(r,t) at different propagation distances z. (a) is the intensity profile for a radius equal to zero of the different temporal snapshots showing the small temporal broadening at $\mathrm{z}=200 \mu \mathrm{m}$. For $\mathrm{z}=200 \mu \mathrm{m}$ the reference pulse (red) as been superposed to the one that has propagated $200 \mu \mathrm{m}$ (green). (b) highlights the presence of a pulse front tilt $\Delta_{\tau}$ visible for $\mathrm{z}=0$. The pulse front tilt is also observed for the different $\mathrm{z}$ positions.

\section{B.Temporal dynamics of SLM based femtosecond Bessel beams}

In section 2, we have shown that the phase profile applied to the pulse by the SLM is the same as the one of an axicon, except that it is wrapped. The spatiotemporal pulse intensity distribution exhibits two important features. First, the pulse produced by an SLM in 
this configuration is $\mathrm{X}$-shaped in space-time representation; it has a superluminal on-axis group velocity. Second, the wrapping operation generates a periodic retardance to the pulse that makes it eventually propagating at sub-luminal speed by averaging on long distance. We detail here this original behavior. To make it clear to the reader, we investigate this effect in both (r, z) in Figure 5 and in $(r, t)$ space in Figure 6. The spatial dispersion $\gamma$, due to the grating structure discussed above, is not considered.

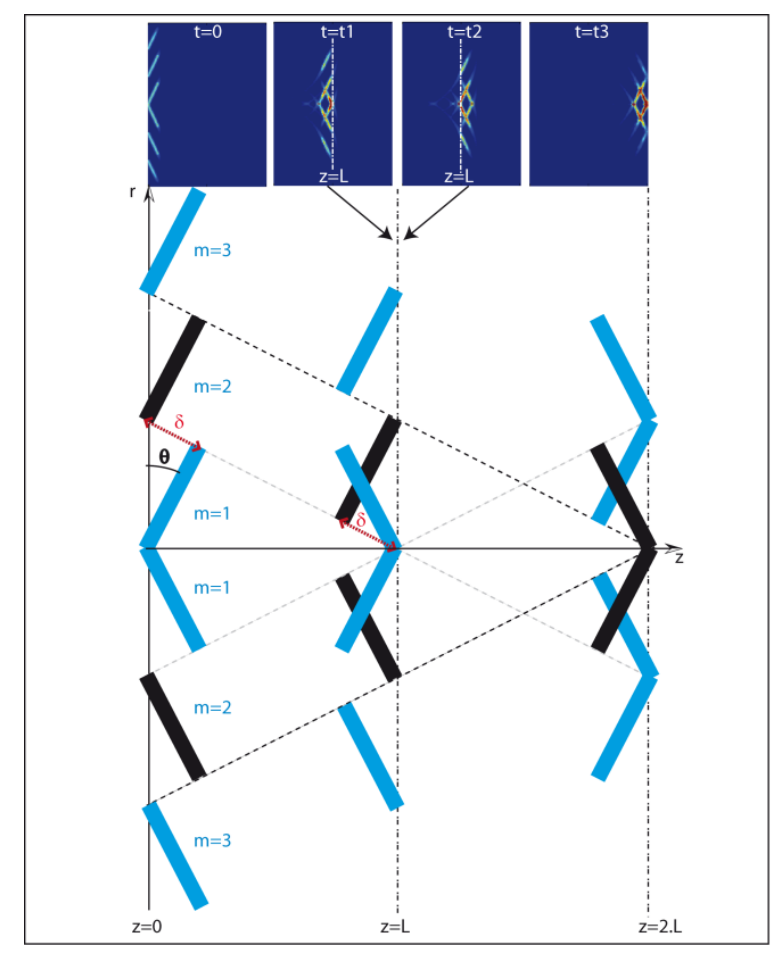

Figure 5. Schematic pulse propagation after the SLM in the $(r, z)$ space. The insets in the upper part of the figure are the different simulated intensity snapshots at different times $t_{0}=0 \quad t_{1}=L \cos \theta / c, t_{2}=t_{2}+\delta / c, t_{3}=2 t_{1}$. 


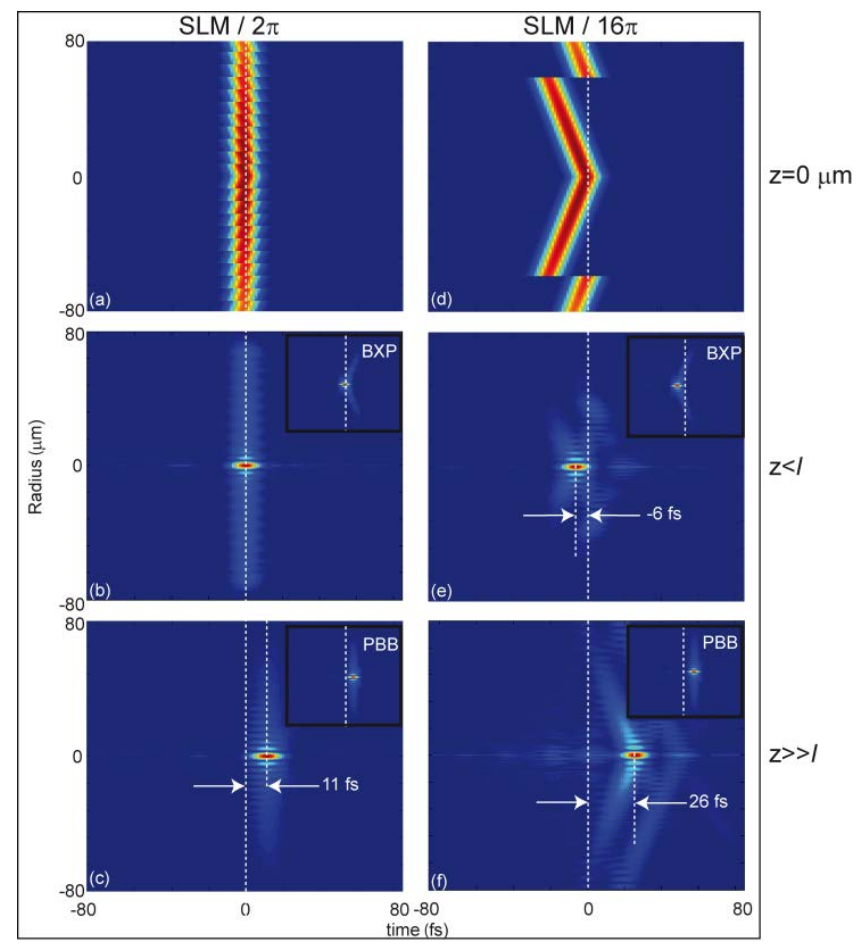

Figure 6. Intensity distribution of a $10 \mathrm{fs}$ pulse in the $(\mathrm{r}, \mathrm{t})$ space at different propagation distances $\mathrm{z}$ after the SLM. The Bessel beam has a conical angle $\theta=5^{\circ}$. Time is expressed in the reference frame of the pulse, moving at speed of light $c$. In the left column (a-b-c), the phase wrapping is performed

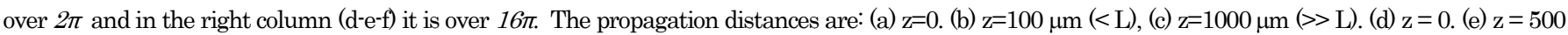
$\mu \mathrm{m}(<\mathrm{L})$. (f) $\mathrm{z}=3000 \mu \mathrm{m}(>>\mathrm{L})$. Note that the value of $\mathrm{L}$ depends on the wrapping ( $\mathrm{N}=2$ or 16).In the insets of each subfigure, we compare with PBB and BXP with the same conical angle and identical propagation distances.

Figure 5 shows schematically in the $(r, z)$ space the propagation of such a pulse at different times. In the insets we plot the corresponding numerical simulations. The phase mask applied at $\mathrm{z}=0$ is initially wrapped over $N \pi$. This produces periodic jumps of the optical path: $\delta=N \lambda_{0} / 2$, that is constant with frequency. The wrapping operation splits the beam into several pairs of subbeams (indexed by $\mathrm{m}=1,2,3 .$. ), shown alternatively in blue and black. Each pair interferes one after the other on the optical axis, over a range $Z=[(m-1) L ; m L]$ where $L=N \lambda_{0} /(2 \sin \theta \tan \theta)$. Here, close to the axis, the pulse is clearly a Bessel X-pulse. To illustrate its superluminal behavior in this propagation range, we plot in figure 6 the results of numerical simulations in $(r, t)$ space at different propagation distances. The left column shows the results for wrapping over $2 \pi$ while on the right, the wrapping is performed over $16 \pi$. The top row shows the pulses at $\mathrm{z}=0$ and the middle row at $\mathrm{z}<\mathrm{L}$. It is clear from the figure that for wrapping over $16 \pi$, the pulse propagates at a superluminal speed $v=c / \cos \theta$ and peaks at negative times $(\Delta t=-6 \mathrm{fs}$ ), while this behavior is obviously less apparent for the case of wrapping over $2 \pi(|\Delta t|<1 \mathrm{fs}$ in our simulations). 
Back to figure 5, we observe at the distance $Z=L$ (and more generally $z_{m}=m L$ ), that the pulse produced on axis by the subbeam $m$ dies when another pulse is generated from subbeam $(m+1)$. Due to the wrapping, this second pulse is generated on axis with a retardance $\delta / c$. This is shown in the insets corresponding to times $t_{2}$ and $t_{3}$ : on the plane $z=L$ (white dashed line), the first pulse dies at $t_{2}$ and another is generated later at $t_{3}$. On average, this reduces the on-axis velocity at a sub-luminal speed $v=c \cos \theta$. Specifically, at a propagation distance $z=m L+d l$, where $d l$ is the residue of $\mathrm{z} / \mathrm{L}$, the relative group delay in the reference frame of the pulse is given by: $\Delta t_{\text {group }}=(m L(1 / \cos \theta-1)+d l \cdot(\cos \theta-1)) / \mathrm{c}$. This behavior is illustrated in the lower row of Figure 6 where we see that for propagation distances $\mathrm{z}>>\mathrm{L}$, the shape of the pulse is closer to a pulsed Bessel beam and the on-axis intensity peak is at positive time in perfect agreement with the expression of $\Delta t_{\text {group }}$ given above. Therefore, the pulse generated by an SLM behaves locally as an $\mathrm{X}$-pulse whereas on long distance, it is close to a pulsed Bessel beam.

\section{Conclusion}

We have numerically investigated the generation of femtosecond Bessel beams with high conical angles with Spatial Light Modulators and shown that the grating-like structure used to separate diffraction orders has a negligible effect on the intensity distribution while it generates pulse front tilt. The investigation of the spatio-temporal structure of SLM-generated Bessel beams shows two distinct features: they behave as Bessel-X pulses at short distance, defined from the wrapping period; and as Pulsed Bessel beams over longer distances, due to the retardance induced by the wrapping operation.

\section{Acknowledgements}

We thank the Région of Franche-Comté and the French ANR, contract 2011-BS04-010-01 NANOFLAM. This work has been performed in cooperation with the Labex ACTION program (contract ANR-11-LABX-01-01).

\section{References}

1. J. Durnin, J. J. Miceli, and J. H. Eberly, "Diffraction-free beams," Phys. Rev. Lett. 58, 1499-1501 (1987).

2. D. Mcgloin, and K. Dholakia, "Bessel beams: Diffraction in a new light," Contemp. Phys. 46, 15-28 (2005).

3. T. Cižmár, V. Kollárová, X. Tsampoula, F. Gunn-Moore, W. Sibbett, Z. Bouchal, and K. Dholakia, "Generation of multiple Bessel beams for a biophotonics workstation,” Opt. Express 16, 14024-14035 (2008).

4. F. O. Fahrbach, P. Simon, and A. Rohrbach, "Microscopy with self-reconstructing beams," Nat. Photonics 4, 780-785 (2010), doi: 10.1038/NPHOTON.2010.204

5. P. Saari and K. Reivelt, "Evidence of X-shaped Propagation-Invariant Localized Light Waves," Physical Review Letters 79, 4135 (1997). 
6. P.Polynkin, M. Kolesik, A. Roberts, D. Faccio, P. Di Trapani, and J. Moloney, "Generation of extended plasma channels in air using femtosecond Bessel beams," Opt. Express 16, 15733-15740 (2008)

7. M. Porras, A. Parola, D. Faccio, A. Dubietis, and P. Di Trapani, "Nonlinear unbalanced Bessel beams: Stationary conical waves supported by nonlinear losses," Phys. Rev. Lett. 93, 153902 (2004).

8. Y. Matsuoka, Y. Kizuka, and T. Inoue, “The characteristics of laser micro drilling using Bessel beam,” Appl. Phys. A 84, 423-430 (2006)

9. M. K. Bhuyan, F. Courvoisier, P.-A. Lacourt, M. Jacquot, L. Furfaro, M. J. Withford, and J. M. Dudley, "High aspect ratio taper-free microchannel fabrication using femtosecond Bessel beams," Opt. Express 18, 566-574 (2010).

10. M. K. Bhuyan, F. Courvoisier, P. A. Lacourt, M. Jacquot, R. Salut, L. Furfaro, and J. M. Dudley, "High aspect ratio nanochannel machining using single shot femtosecond Bessel beams,” Appl. Phys. Lett. 97, 081102 (2010).

11. M.A. Porras, P. Di Trapani and W. Hu,"Optical Wave Modes : Localized and Propagation-Invariant Wave Packets in Optically Transparent Dispersive Media", H.E. Hernandez-Figueroa, M. Zamboni-Rached, E. Recami, eds. (Wiley, 2008), pp. 217-241.

12. M. Clerici, D. Faccio, A. Lotti, E. Rubino, O. Jedrkiewicz, J. Biegert, and P. Di Trapani, "Finite-energy, accelerating Bessel pulses," Opt. Express 16, 19807-19811 (2008).

13. C. Sheppard, "Bessel pulse beams and focus wave modes," J. Opt. Soc. Am. A 18, 2594-2600 (2001).

14. A. Vasara, J. Turunen, and A. T. Friberg "Realization of general nondiffracting beams with computer-generated holograms," J. Opt. Soc. Am. A 6, 1748-1754 (1989)

15. M. Bock, S. K. Das, and R. Grunwald, "Programmable ultrashort-pulsed flying images," Opt. Express 17, 7465-7478 (2009) .

16. N. Chattrapiban, E. A. Rogers, D. Cofield, W. T. Hill, and R. Roy, "Generation of nondiffracting Bessel beams by use of a spatial light modulator" Opt. Lett., 28, 2183-2185 (2003).

17. M. Bock, S. K. Das, and R. Grunwald, "Ultrashort highly localized wavepackets", Opt. Express 20, 12563-12578 (2012);

18. M. Bock, S. K. Das, C. Fischer, M. Diehl, P. Börner, and R. Grunwald, "Reconfigurable wavefront sensor for ultrashort pulses", Opt. Lett. 37, 1154-1156 (2012).

19. T. Hara "A liquid crystal spatial light phase modulator and its applications", Proc. SPIE 5642, 78-89 (2005).

20. J. Leach, G. M. Gibson, M. J. Padgett, E. Esposito, G. McConnell, A. J. Wright, and J. M. Girkin, "Generation of achromatic Bessel beams using a compensated spatial light modulator," Opt. Express 14, 5581-5587 (2006).

21. J. W. Goodman, Introduction to Fourier Optics (McGraw Hill, 1996).

22. A.M. Weiner, "Ultrafast Optics” (Wiley Series in Pure and Applied Optics, 2009) 